\title{
Development of a Hybrid Prediction Mechanism using SMA and EXS Methods for GSM Logical Channel Load Variables
}

\author{
Garba S. \\ Department of Electrical and \\ Computer Engineering, Ahmadu \\ Bello University, Zaria.
}

\author{
Mu'azu M.B. \\ Department of Electrical and \\ Computer Engineering, Ahmadu \\ Bello University, Zaria.
}

\author{
Dajab D.D. \\ Department of Electrical and \\ Computer Engineering, Ahmadu \\ Bello University, Zaria.
}

\begin{abstract}
The GSM logical channel load are stochastic (random), distinct in time (Erlang) distribution data; and as such it requires robust means of its prediction. The method employed in this work for the predictions is a hybrid of Simple Moving Average (SMA) and Exponential Smoothing (ExS), which can fit in to predict logical channel load variables with it peculiarities. A three (3) month Data were used in determining the number of observations for the prediction (n) for SMA and smoothing constant $(\alpha)$ for ExS. The determinant values obtained are $\mathrm{n}=28$, and $\alpha=0.077$. These values are used to predict the logical control and traffic channels load variables that characterizes its utilization.
\end{abstract}

\section{Keywords}

GSM, SMA, ExS, and Logical channel.

\section{INTRODUCTION}

In a GSM system, the physical channels carry different messages, depending on the information that is to be sent. These messages are carried via channels called logical channels (traffic and control). The logical channels loading impact on the network due to the nature of its variability. These include Control Channel Drop Calls rate (PCDROP), Control Channel Congestion rate (PCCONG), Control Channel Failure rate (PCFAIL), Traffic Channel Congestion rate (PCONG), Traffic Channel Availability rate (PTTCH), and Traffic Channel Failure rate (PTFAIL) [1]. These channel variables (data generated) are presented in Figures 1 and 2, based on Appendix A1, to identify their characteristics and nature.

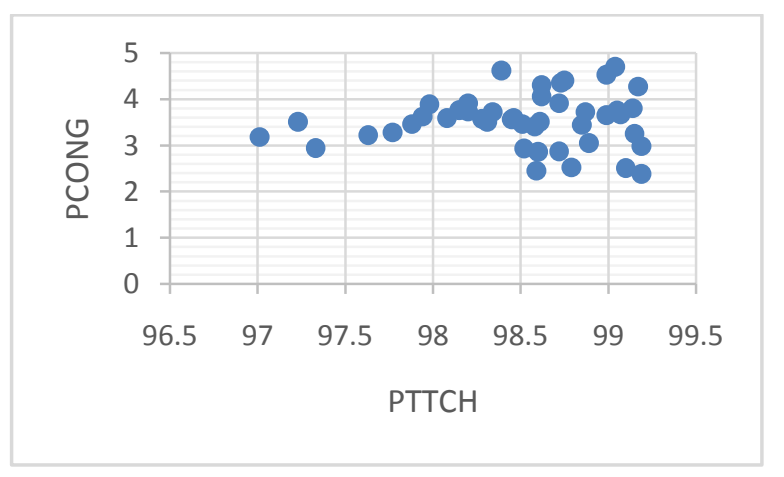

Figure 1: Plots of PCONG Vs PTTCH

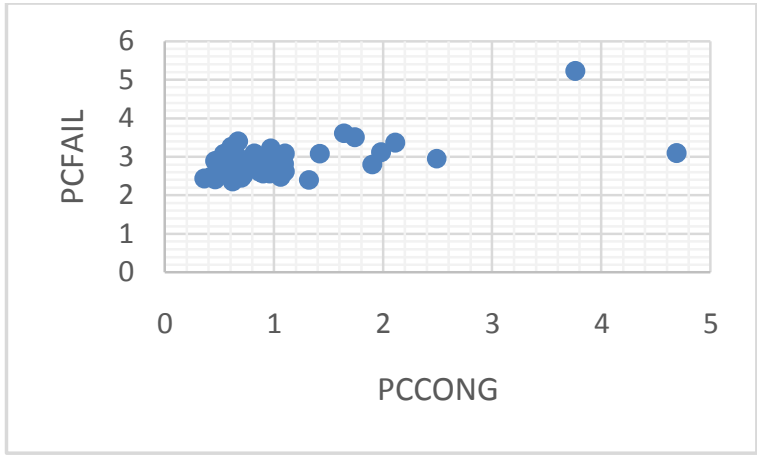

Figure 2: Plots of PCFAIL Vs PCCONG

From the plots presented, the variations are observed to be scattered, which shows that the generated data are stochastic (random) in nature and distinct in time distribution (stochastic). Stochastic variables belong to a family of variables that are non-linear, uncertain, and random [2].

These stochastic variables analyses relies in exploiting the dependence among the channel variables. And the logical channel variables (control and traffic) are distinct in time, which indicates that their analysis can be achieved using Erlang distribution [3]. The Erlang measures the average occupancy of traffic route over a period in communications. It is a dimensionless quantity, and measures to certain extent carried traffic on the network. The Erlang distribution equation is given by [4]:

$$
p=\frac{\left(y^{n} / n !\right)}{\left[\sum_{x=0}^{n}\left(y^{n} / n !\right)\right]}
$$

Where, $\quad \mathbf{p}=$ probability of loss or blocking

$$
\begin{aligned}
& \mathbf{n}=\text { number of paths } \\
& \mathbf{y}=\text { traffic offered (Erlang) }
\end{aligned}
$$

Equation (1) can further be expanded to:

$$
p=\frac{\left(\begin{array}{c}
N \\
m
\end{array}\right)\left(\begin{array}{c}
\lambda_{o} \\
\mu
\end{array}\right)^{m}}{\sum_{v=0}^{\mathrm{m}}\left(\begin{array}{c}
N \\
m
\end{array}\right)\left(\begin{array}{c}
\lambda_{o} \\
\mu
\end{array}\right)^{v}}
$$

Where, $\mathbf{N}=$ system capacity

$$
\begin{aligned}
& \mathbf{m}=\text { number of channels/circuits } \\
& \lambda_{\boldsymbol{o}}=\text { threshold traffic intensity } \\
& \boldsymbol{\mu}=\text { service rate } \\
& \mathbf{v}=\text { number of busy channels at time } \mathrm{t}
\end{aligned}
$$


In stochastic variables prediction, known values of the variables are needed up to the point in time, say, $t$, to predict the value at some point in the future, say, $t+P$. The standard method for this type of prediction is to create a mapping from D sample data points, sampled every $\Delta$ units in time (x(t-(D) 1) $\Delta), \ldots, \mathbf{x}(\mathbf{t}-\Delta), \mathbf{x}(\mathbf{t}))$, to a predicted future value $\mathbf{x}(\mathbf{t}+\mathbf{P})$ [5].

\section{METHODOLOGY}

The method employed in finding an optimal continuation of the GSM logical channel load variables is a hybrid of the Simple Moving Average (SMA) and Exponential Smoothing $(\mathrm{ExS})$ methods. The sequence involved in achieving the optimal hybrid involves the determinations of:

$$
\begin{array}{ll}
\text { i. } & \text { Data set mean }\left(\mathrm{x}_{\mathrm{i}}\right) ; \\
\text { ii. } & \text { Number of observations }(\mathrm{n}) \text {; and } \\
\text { iii. } & \text { Smoothening constant }(\alpha) .
\end{array}
$$

\subsection{Simple Moving Averages (SMA)}

SMA is the averaging of the absolute deviations of series of data above from their mean. Equally, it is a technique that uses a type of average that is adjusted to allow for seasonal, cyclical or stochastic variables of a series data and brings either short, mid-, long term trends clearer. In SMA only $\mathbf{n}$ most recent periods of data points need to be maintained. At the end of each sequence of $\mathbf{n}$, the oldest sequence is discarded and the newest data is added to the database. The database is then divided by $\mathbf{n}$ and used as a forecast for the next period [6], [7].

The moving average $\mathbf{x}(\mathbf{t}+\mathbf{1})$ for stochastic data of a period ( $\mathbf{t}$ +1 ) is given by:

$$
x(t+1)=\frac{\left[D_{1}+D_{t-1}+\cdots+D_{t-n-1}\right]}{n},
$$

$n \leq t$

Where, $\mathbf{n}=$ number of observations used

$$
\mathbf{D}=\text { stochastic data }
$$

\subsection{Determination of Number of Observations (n)}

The number of observations (n) is determined by selecting a value that minimizes the Mean Square Error (MSE) of prediction, given by [6]:

$M S E=\sum_{i=1}^{n} \frac{\left[\left(D_{i}-x_{i}\right)\right]^{2}}{n}$

Where, $\boldsymbol{x}_{\boldsymbol{i}}=$ mean of the data set

The value $\boldsymbol{x}_{\boldsymbol{i}}$ was obtained using a moving window of fourteen (14)-day, twenty one (21)-day, and twenty eight (28)day width $(\mathbf{n}=14, \mathbf{n}=21$, and $\mathbf{n}=28)$ on logical channels load variables. The computed $\boldsymbol{x}_{\boldsymbol{i}}$ is shown in Table 1 .

Table 1: $x_{i}$ values for logical channels variables

\begin{tabular}{|l|l|l|l|l|l|l|}
\hline $\mathbf{n}$ & PTTCH & PCONG & PTFAIL & PCDROP & PCCONG & PCFAIL \\
\hline 14 & 98.45 & 3.1 & 1.09 & 0.37 & 0.82 & 2.57 \\
\hline 21 & 98.41 & 3.12 & 1.11 & 0.38 & 0.87 & 2.56 \\
\hline 28 & 98.42 & 3.15 & 1.11 & 0.37 & 0.84 & 2.62 \\
\hline
\end{tabular}

\begin{tabular}{|c|c|c|c|c|c|c|c|c|c|}
\hline \multirow{3}{*}{$\begin{array}{l}\text { Input } \\
\text { variables }\end{array}$} & \multicolumn{3}{|c|}{ January, 2010} & \multicolumn{3}{|c|}{ February, 2010} & \multicolumn{3}{|c|}{ March, 2010} \\
\hline & \multicolumn{3}{|l|}{$\mathbf{n}$} & \multicolumn{3}{|l|}{$\mathbf{n}$} & \multicolumn{3}{|l|}{$\mathbf{n}$} \\
\hline & 14 & 21 & 28 & 14 & 21 & 28 & 14 & 21 & 28 \\
\hline PITCH & 0.3219 & 0.3170 & 0.3179 & 0.2146 & 0.2160 & 0.2103 & 0.5323 & 0.5299 & 0.5302 \\
\hline PCONG & 0.4610 & 0.4610 & 0.4610 & 0.5411 & 0,5401 & 0.5401 & 0.3936 & 0.3926 & 0.3927 \\
\hline PTFAIL & 0.0105 & 0.0134 & 0.0134 & 0.0048 & 0.0062 & 0.0062 & 0.0058 & 0.0053 & 0.0053 \\
\hline PCDROP & 0.0025 & 0.0035 & 0.0025 & 0.0029 & 0.0040 & 0.0029 & 0.0007 & 0.0009 & 0.0007 \\
\hline PCCONG & 1.0604 & 1.0217 & 1.0444 & 0.4246 & 0.4186 & 0.4216 & 0.108 & 0.1148 & 0.1101 \\
\hline PCFAIL & 0.4296 & 0.4388 & 0.3866 & 0.3426 & 0.3458 & 0.3295 & 0.0659 & 0.0671 & 0.0631 \\
\hline
\end{tabular}

Subsequently $\boldsymbol{x}_{\boldsymbol{i}}$ was applied to the validation data on Appendix A1, for the choice of $\mathbf{n}$ to minimize the Mean Square Error (MSE). The result is shown in Table 2.

Table 2: MSE values for $\mathbf{n}$

From Table 2, $\mathbf{n}=\mathbf{2 8}$ generate the least Mean Square Error (MSE) value, therefore it was chosen. The selected value of $\mathbf{n}$ is then used to determine the forecast values for the logical control and traffic channels variables from April to June, 2010.

\subsection{Exponential Smoothing Method (ExS)}

ExS is a forecasting technique that weighs past data in an exponential manner, so that the most recent data carries more weight. The exponentially smoothed moving average is based not on a sequential average of individual stochastic periods, but on the most recent data and the average prior to it adjusted by a smoothing constant, $\alpha$. The expression for the smoothing constant is [6], [8]: 


$$
\alpha=\frac{1}{\frac{n}{2}-1}
$$

The smoothed series is updated as new observations are recorded using the expression:

$$
S(t)=\alpha \mathrm{D}(\mathbf{t})+(\mathbf{1}-\boldsymbol{\alpha}) \mathbf{S}(\mathbf{t}-\mathbf{1})
$$

Where, $\mathrm{S}(\mathrm{t})$ is the value of the smoothed stochastic series.

The current smoothed value $S(t)$ is an interpolation between previous smoothed value $(\mathrm{S}(\mathrm{t}-1))$ and the current observation, and $\alpha$ controls the closeness of the interpolated value of the recent observation [6].

The complete forecasted values of the logical control and traffic channels variables using the hybrid of Moving Averages and Exponential Smoothing methods is shown in Appendix A2. These values are used to plot the logical control and traffic channels load variables for the next three (3) months. The plots for Control Channel Drop Calls rate (PCDROP); Control Channel Congestion rate (PCCONG); Control Channel Failure rate (PCFAIL); Traffic Channel Congestion rate (PCONG); Traffic Channel Availability rate (PTTCH); and Traffic Channel Failure (PTFAIL) are shown in Figures 3 to 8.

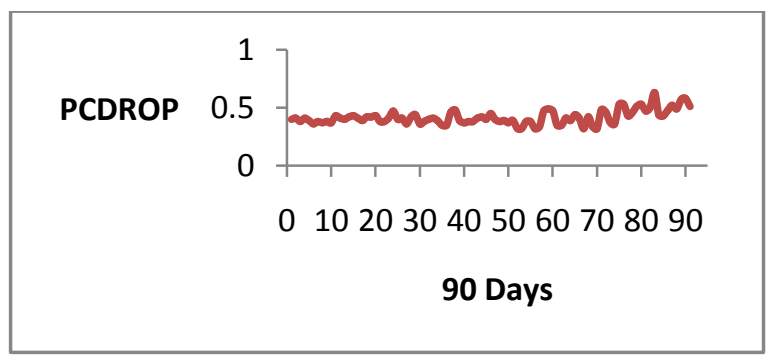

Figure 3: Plot of Control Channels Call Drop rate (PCDROP)

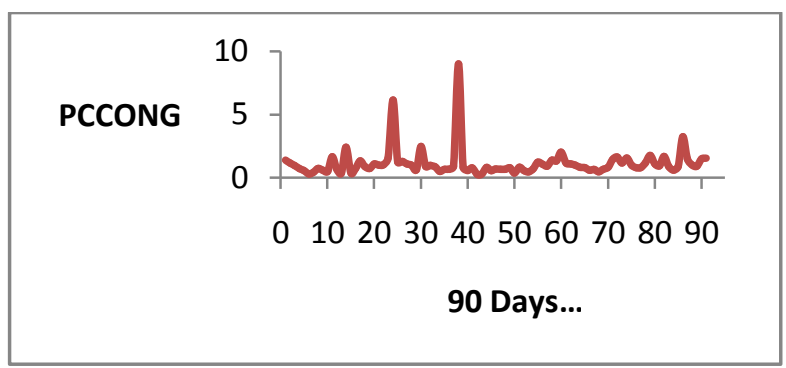

Figure 4: Plot of Control Channels Congestion rate (PCCONG).

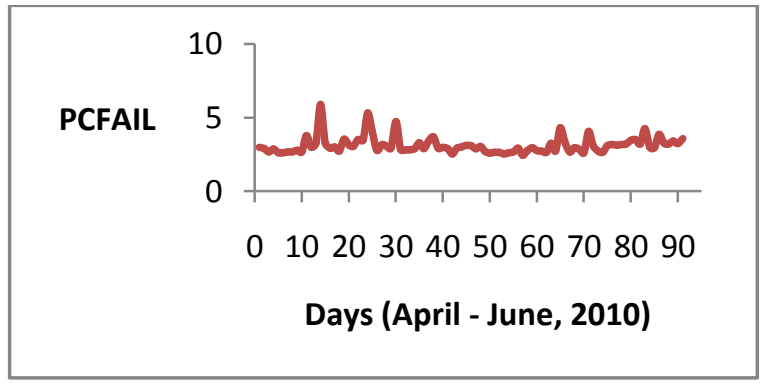

Figure 5: Plot of Control Channels Failure rate (PTFAIL).

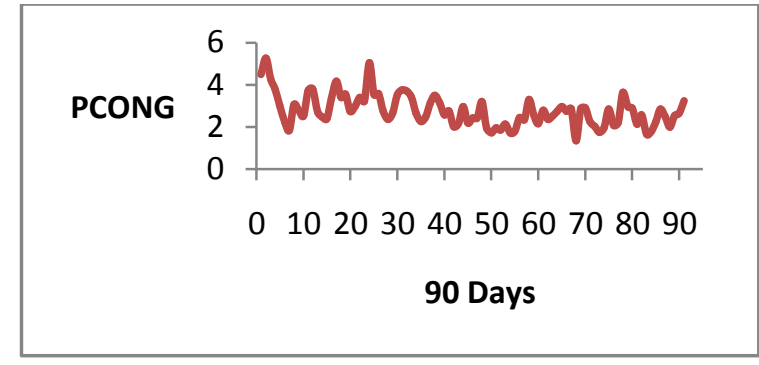

Figure 6: Plot of Traffic Channels Congestion rate (PCONG).

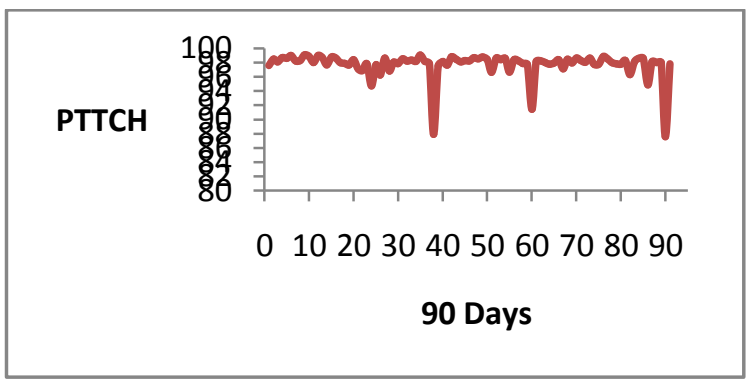

Figure 7: Plot of Traffic Channels Availability rate (PTTCH).

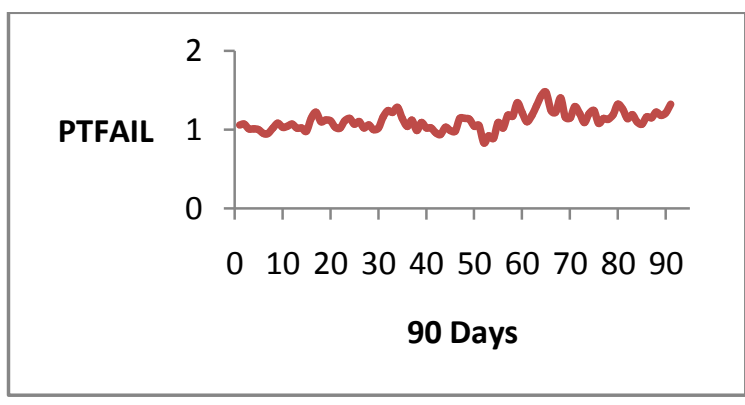

Figure 8: Plot of Traffic Channels Failure rate

\section{DISCUSSION OF RESULTS}

The forecasted results in Appendix A2, which is represented in Figures 4-9 demonstrate the viability of the mechanism (hybrid of SMA and ExS methods) for stochastic forecasting. The main features of the results (shown in Figures 1-4) is the ability of the mechanism to convey constant closeness control and smoothening of previous and current observations. This is achieved based on consistent interpolation of previous and current observations.

\section{CONCLUSIONS}

This paper presents a method for prediction of stochastic (random), and distinct in time (Erlang) distribution data. The method employed is a hybrid of Simple Moving Average and Exponential Smoothing. The data used is the GSM logical (control and traffic) channel load variables which is stochastic in nature and followed the Erlang distribution pattern.

The developed hybrid method has demonstrated that it can be used to forecast for GSM logical control channel variables (call drop, failure, and congestion rates) and traffic channel variables (congestion, availability, and failure rates) of a GSM radio network. One of its major features is mapping and sampling of data points and units, weighting of past data, and sequential averaging. 


\section{REFERENCES}

[1] Sulaiman A. T. 2009. RF Network Fault Analysis of MTN Kano Region. M.Sc Thesis. Department of Electrical Engineering, A B U, Zaria. 4-38.

[2] Poularikas A. D. 1999. Probability and Stochastic Processes. The Handbook of Formulas and Tables for Signal Processing. Ed. Alexander D. Poularikas Boca Raton: CRC Press LLC. 41-52.

[3] Kennedy G., \& Davis B. 1993. Electronic Communication Systems. New Delhi: Tata McGraw-Hill Publishing Company Ltd. 593-595.

[4] ITU. 2002. Handbook on Satellite Communications. U.S: Wiley Interscience, a John Wiley and Sons, Inc. Publication. 344-345.
[5] MATLAB® V7.9.0.529. 2009. Neuro-fuzzy computing based on fuzzy logic toolbox. MATLAB works (R2009b)

[6] Mu'azu, M.B. 2006. Forecasting and modeling statistical phenomena using neuro-fuzzy logic: A case study of rainfall forecasting for Zaria. $\mathrm{PhD}$ Dissertation. Department of Electrical Engineering, A B U, Zaria. 2, 95-105, 117-120, 134-136.

[7] Monks, J. G. 1996. Schaum's outline of theory and Problems of Operations Management, $2^{\text {nd }}$ edition, McGraw-Hill inc. 39-43.

[8] Average and Exponential Smoothing Models (Decision 411 Forecasting). Retrieved August 7, 2010 from www.duke.edu/mau/411avg.htm

Appendix A1: Validation data

\begin{tabular}{|c|c|c|c|c|c|c|c|c|c|c|c|c|c|}
\hline DISPLAY & $\begin{array}{l}\text { PTTC } \\
\text { H }\end{array}$ & $\begin{array}{l}\text { PCON } \\
\text { G }\end{array}$ & $\begin{array}{l}\text { PTFAI } \\
\text { L }\end{array}$ & $\begin{array}{l}\text { PCDR } \\
\text { OP }\end{array}$ & $\begin{array}{l}\text { PCCO } \\
\text { NG }\end{array}$ & $\begin{array}{l}\text { PCFAI } \\
\text { L }\end{array}$ & DISPLAY & $\begin{array}{l}\text { PTT } \\
\text { CH }\end{array}$ & $\begin{array}{l}\text { PCO } \\
\text { NG }\end{array}$ & $\begin{array}{l}\text { PTF } \\
\text { AIL }\end{array}$ & $\begin{array}{l}\text { PCD } \\
\text { ROP }\end{array}$ & $\begin{array}{l}\text { PCC } \\
\text { ONG }\end{array}$ & $\begin{array}{l}\text { PCF } \\
\text { AIL }\end{array}$ \\
\hline 2010-01-01 & 99.04 & 4.7 & 1.17 & 0.33 & 1.01 & 2.96 & $2010-02-15$ & 98.78 & 2.59 & 1.01 & 0.32 & 0.56 & 2.49 \\
\hline 2010-01-02 & 99.17 & 4.27 & 1.13 & 0.31 & 0.86 & 2.79 & $2010-02-16$ & 99.01 & 2.14 & 1.03 & 0.32 & 0.69 & 2.48 \\
\hline $2010-01-03$ & 99.05 & 3.75 & 1.17 & 0.32 & 0.63 & 2.85 & $2010-02-17$ & 98.91 & 2.47 & 1.07 & 0.32 & 0.96 & 2.5 \\
\hline 2010-01-04 & 98.87 & 3.72 & 1.21 & 0.32 & 0.75 & 2.79 & $2010-02-18$ & 98.86 & 2.15 & 0.99 & 0.3 & 0.76 & 2.37 \\
\hline 2010-01-05 & 98.34 & 3.72 & 1.19 & 0.32 & 0.56 & 2.86 & $2010-02-19$ & 98.94 & 2.36 & 1.03 & 0.32 & 0.69 & 2.47 \\
\hline 2010-01-06 & 98.28 & 3.57 & 1.16 & 0.32 & 0.63 & 2.7 & $2010-02-20$ & 97.81 & 3.49 & 1.12 & 0.35 & 1.75 & 2.61 \\
\hline $2010-01-07$ & 97.88 & 3.46 & 1.13 & 0.31 & 0.46 & 2.89 & $2010-02-21$ & 97.99 & 2.86 & 1.08 & 0.34 & 0.77 & 2.69 \\
\hline 2010-01-08 & 98.45 & 3.56 & 1.12 & 0.32 & 0.66 & 2.92 & $2010-02-22$ & 92.76 & 3.77 & 1.04 & 0.37 & 3.72 & 2.47 \\
\hline 2010-01-09 & 98.2 & 3.91 & 1.02 & 0.34 & 0.82 & 3.09 & $2010-02-23$ & 98.78 & 2.42 & 1.05 & 0.33 & 0.59 & 3.81 \\
\hline 2010-01-10 & 97.01 & 3.18 & 0.98 & 0.39 & 1.9 & 2.8 & $2010-02-24$ & 98.51 & 2.27 & 1.13 & 0.34 & 0.31 & 2.82 \\
\hline 2010-01-11 & 98.89 & 3.05 & 0.96 & 0.33 & 0.61 & 3.26 & $2010-02-25$ & 98.56 & 4.47 & 1.09 & 0.35 & 0.63 & 2.89 \\
\hline 2010-01-12 & 97.63 & 3.22 & 0.99 & 0.3 & 4.69 & 3.1 & $2010-02-26$ & 98.93 & 3.31 & 1.22 & 0.31 & 0.67 & 2.54 \\
\hline 2010-01-13 & 98.46 & 3.59 & 0.94 & 0.31 & 0.54 & 3.08 & $2010-02-27$ & 98.95 & 2.67 & 1.2 & 0.31 & 0.37 & 2.58 \\
\hline 2010-01-14 & 98.52 & 2.93 & 0.97 & 0.32 & 0.88 & 2.84 & $2010-02-28$ & 98.48 & 3.42 & 1.25 & 0.33 & 1.48 & 5.13 \\
\hline 2010-01-15 & 98.51 & 3.46 & 1.01 & 0.36 & 1.98 & 3.12 & 2010-03-01 & 98.85 & 3.07 & 1.13 & 0.32 & 0.6 & 2.66 \\
\hline $2010-01-16$ & 97.23 & 3.51 & 1.01 & 0.33 & 1.42 & 3.08 & $2010-03-02$ & 97.91 & 2.96 & 1.12 & 0.33 & 0.49 & 2.76 \\
\hline 2010-01-17 & 98.15 & 3.76 & 0.93 & 0.28 & 0.82 & 3.04 & $2010-03-03$ & 99.06 & 2.8 & 1.18 & 0.34 & 0.4 & 2.53 \\
\hline 2010-01-18 & 97.33 & 2.94 & 0.95 & 0.3 & 0.9 & 2.57 & 2010-03-04 & 98.91 & 2.89 & 1.16 & 0.34 & 0.44 & 2.56 \\
\hline 2010-01-19 & 97.98 & 3.89 & 1.02 & 0.31 & 3.76 & 5.23 & $2010-03-05$ & 98.29 & 3.69 & 1.19 & 0.37 & 0.57 & 2.75 \\
\hline $2010-01-20$ & 98.2 & 3.73 & 0.94 & 0.29 & 1.32 & 2.4 & 2010-03-06 & 97.51 & 3.97 & 1.17 & 0.38 & 0.78 & 3.21 \\
\hline $2010-01-21$ & 98.6 & 2.86 & 0.95 & 0.32 & 0.66 & 3.01 & 2010-03-07 & 97.02 & 3.82 & 1.15 & 0.36 & 1.09 & 3.46 \\
\hline $2010-01-22$ & 97.77 & 3.28 & 0.98 & 0.34 & 2.49 & 2.95 & 2010-03-08 & 98.55 & 2.78 & 1.07 & 0.35 & 0.3 & 2.72 \\
\hline $2010-01-23$ & 99.14 & 3.8 & 1 & 0.34 & 1.1 & 3.09 & 2010-03-09 & 98.68 & 2.81 & 1.02 & 0.31 & 0.71 & 2.37 \\
\hline $2010-01-24$ & 98.58 & 3.41 & 1.03 & 0.34 & 1.09 & 2.82 & 2010-03-10 & 99.01 & 2.83 & 1.07 & 0.37 & 0.53 & 2.45 \\
\hline $2010-01-25$ & 98.72 & 2.87 & 0.99 & 0.33 & 1.74 & 3.51 & $2010-03-11$ & 96.82 & 1.9 & 1.11 & 0.37 & 1.33 & 2.34 \\
\hline $2010-01-26$ & 99.07 & 3.67 & 1.01 & 0.32 & 0.67 & 3.4 & 2010-03-12 & 97.1 & 4.55 & 1.29 & 0.41 & 1.1 & 2.47 \\
\hline 2010-01-27 & 98.31 & 3.51 & 0.96 & 0.35 & 0.97 & 3.22 & 2010-03-13 & 98.81 & 4.63 & 1.29 & 0.42 & 1.34 & 2.66 \\
\hline $2010-01-28$ & 98.72 & 3.91 & 1.02 & 0.32 & 2.11 & 3.37 & $2010-03-14$ & 99.09 & 3.23 & 1.12 & 0.37 & 0.77 & 2.61 \\
\hline $2010-01-29$ & 98.99 & 4.53 & 1.01 & 0.33 & 0.6 & 2.79 & $2010-03-15$ & 98.82 & 3.18 & 1.11 & 0.41 & 0.78 & 2.52 \\
\hline 2010-01-30 & 97.94 & 3.62 & 0.97 & 0.33 & 0.57 & 2.66 & $2010-03-16$ & 98.74 & 3.08 & 1.1 & 0.38 & 0.88 & 3.01 \\
\hline 2010-01-31 & 98.39 & 4.62 & 0.98 & 0.32 & 0.99 & 2.6 & 2010-03-17 & 98.96 & 1.51 & 1.03 & 0.34 & 0.5 & 2.31 \\
\hline $2010-02-01$ & 98.62 & 4.3 & 1.08 & 0.33 & 1.07 & 2.74 & $2010-03-18$ & 98.19 & 2.92 & 1.07 & 0.32 & 0.5 & 2.3 \\
\hline 2010-02-02 & 98.73 & 4.35 & 1.08 & 0.34 & 0.72 & 2.53 & 2010-03-19 & 96.91 & 3.39 & 1.2 & 0.35 & 1.86 & 2.49 \\
\hline
\end{tabular}




\begin{tabular}{|c|c|c|c|c|c|c|c|c|c|c|c|c|c|}
\hline $2010-02-03$ & 98.75 & 4.4 & 1.03 & 0.3 & 0.7 & 2.5 & $2010-03-20$ & 98.52 & 3.12 & 1.19 & 0.37 & 0.68 & 2.97 \\
\hline $2010-02-04$ & 98.08 & 3.59 & 1.04 & 0.29 & 1.1 & 2.62 & $2010-03-21$ & 98.89 & 3.35 & 1.13 & 0.36 & 0.78 & 2.58 \\
\hline $2010-02-05$ & 98.61 & 3.51 & 0.96 & 0.29 & 0.62 & 2.36 & $2010-03-22$ & 99 & 3.24 & 1.09 & 0.39 & 0.73 & 2.57 \\
\hline 2010-02-06 & 99.15 & 3.25 & 1 & 0.29 & 1.06 & 2.48 & $2010-03-23$ & 98.31 & 2.77 & 1.09 & 0.39 & 0.68 & 2.65 \\
\hline $2010-02-07$ & 98.85 & 3.44 & 1.03 & 0.31 & 0.87 & 2.61 & $2010-03-24$ & 99.09 & 2.59 & 1.07 & 0.37 & 0.48 & 2.5 \\
\hline 2010-02-08 & 98.99 & 3.65 & 1.02 & 0.29 & 0.46 & 2.41 & $2010-03-25$ & 98.68 & 2.87 & 1.01 & 0.36 & 0.51 & 2.51 \\
\hline $2010-02-09$ & 98.59 & 2.45 & 1.04 & 0.33 & 0.36 & 2.44 & $2010-03-26$ & 98.75 & 3.81 & 1.11 & 0.38 & 0.95 & 2.55 \\
\hline $2010-02-10$ & 99.1 & 2.51 & 1.03 & 0.31 & 0.7 & 2.46 & $2010-03-27$ & 96.93 & 3.48 & 1.09 & 0.38 & 0.95 & 2.45 \\
\hline $2010-02-11$ & 98.79 & 2.52 & 1.04 & 0.3 & 0.43 & 2.49 & $2010-03-28$ & 98.73 & 3.25 & 1.1 & 0.37 & 1.09 & 2.47 \\
\hline 2010-02-12 & 99.19 & 2.98 & 1.06 & 0.33 & 0.71 & 2.64 & 2010-03-29 & 99.28 & 2.95 & 1.06 & 0.36 & 0.55 & 2.42 \\
\hline $2010-02-13$ & 99.19 & 2.38 & 1.06 & 0.34 & 1.64 & 3.61 & $2010-03-30$ & 98.35 & 3.23 & 1.04 & 0.39 & 0.96 & 2.72 \\
\hline $2010-02-14$ & 98.62 & 4.06 & 1.09 & 0.31 & 0.96 & 2.57 & $2010-03-31$ & 98.64 & 2.47 & 0.95 & 0.38 & 0.76 & 2.73 \\
\hline
\end{tabular}

Appendix A2: Forecast results of Moving averages and Exponential smoothing methods

\begin{tabular}{|r|r|r|l|r|l|l|}
\hline \multicolumn{7}{|c|}{ PCONG FORECAST TABLE $(\mathbf{n}=\mathbf{2 8}$ and $\boldsymbol{\alpha}=\mathbf{0 . 0 7 7})$} \\
\hline DAY & APRIL & & \multicolumn{1}{|l|}{ MAY } & & JUNE & \\
\hline $\mathbf{1}$ & 4.49 & $\mathbf{4 . 4 9}$ & 3.53771 & $\mathbf{3 . 7 5}$ & 2.75766 & $\mathbf{2 . 3 7}$ \\
\hline $\mathbf{2}$ & 4.54929 & $\mathbf{5 . 2 6}$ & 3.74615 & $\mathbf{3 . 7}$ & 2.38155 & $\mathbf{2 . 5 2}$ \\
\hline $\mathbf{3}$ & 5.18531 & $\mathbf{4 . 2 9}$ & 3.67767 & $\mathbf{3 . 4 1}$ & 2.53694 & $\mathbf{2 . 7 4}$ \\
\hline $\mathbf{4}$ & 4.25073 & $\mathbf{3 . 7 8}$ & 3.35148 & $\mathbf{2 . 6 5}$ & 2.75771 & $\mathbf{2 . 9 7}$ \\
\hline $\mathbf{5}$ & 3.72071 & $\mathbf{3 . 0 1}$ & 2.61997 & $\mathbf{2 . 2 6}$ & 2.95306 & $\mathbf{2 . 7 5}$ \\
\hline $\mathbf{6}$ & 2.95071 & $\mathbf{2 . 2 4}$ & 2.27694 & $\mathbf{2 . 4 8}$ & 2.75924 & $\mathbf{2 . 8 7}$ \\
\hline $\mathbf{7}$ & 2.2092 & $\mathbf{1 . 8 4}$ & 2.53005 & $\mathbf{3 . 1 3}$ & 2.75296 & $\mathbf{1 . 3 5}$ \\
\hline $\mathbf{8}$ & 1.93394 & $\mathbf{3 . 0 6}$ & 3.15849 & $\mathbf{3 . 5}$ & 1.46704 & $\mathbf{2 . 8 7}$ \\
\hline $\mathbf{9}$ & 3.04075 & $\mathbf{2 . 8 1}$ & 3.47074 & $\mathbf{3 . 1 2}$ & 2.87308 & $\mathbf{2 . 9 1}$ \\
\hline $\mathbf{1 0}$ & 2.78767 & $\mathbf{2 . 5 2}$ & 3.07765 & $\mathbf{2 . 5 7}$ & 2.85995 & $\mathbf{2 . 2 6}$ \\
\hline $\mathbf{1 1}$ & 2.6124 & $\mathbf{3 . 7 2}$ & 2.58386 & $\mathbf{2 . 7 5}$ & 2.24152 & $\mathbf{2 . 0 2}$ \\
\hline $\mathbf{1 2}$ & 3.72616 & $\mathbf{3 . 8}$ & 2.69379 & $\mathbf{2 . 0 2}$ & 1.99921 & $\mathbf{1 . 7 5}$ \\
\hline $\mathbf{1 3}$ & 3.71607 & $\mathbf{2 . 7 1}$ & 2.03232 & $\mathbf{2 . 1 8}$ & 1.76694 & $\mathbf{1 . 9 7}$ \\
\hline $\mathbf{1 4}$ & 2.69152 & $\mathbf{2 . 4 7}$ & 2.24083 & $\mathbf{2 . 9 7}$ & 2.03776 & $\mathbf{2 . 8 5}$ \\
\hline $\mathbf{1 5}$ & 2.46384 & $\mathbf{2 . 3 9}$ & 2.90994 & $\mathbf{2 . 1 9}$ & 2.79071 & $\mathbf{2 . 0 8}$ \\
\hline $\mathbf{1 6}$ & 2.46546 & $\mathbf{3 . 3 7}$ & 2.20848 & $\mathbf{2 . 4 3}$ & 2.09001 & $\mathbf{2 . 2 1}$ \\
\hline $\mathbf{1 7}$ & 3.4316 & $\mathbf{4 . 1 7}$ & 2.43077 & $\mathbf{2 . 4 4}$ & 2.32011 & $\mathbf{3 . 6 4}$ \\
\hline $\mathbf{1 8}$ & 4.11071 & $\mathbf{3 . 4 0}$ & 2.49929 & $\mathbf{3 . 2 1}$ & 3.58764 & $\mathbf{2 . 9 6}$ \\
\hline $\mathbf{1 9}$ & 3.41155 & $\mathbf{3 . 5 5}$ & 3.11375 & $\mathbf{1 . 9 6}$ & 2.95384 & $\mathbf{2 . 8 8}$ \\
\hline $\mathbf{2 0}$ & 3.48763 & $\mathbf{2 . 7 4}$ & 1.94152 & $\mathbf{1 . 7 2}$ & 2.82302 & $\mathbf{2 . 1 4}$ \\
\hline $\mathbf{2 1}$ & 2.75925 & $\mathbf{2 . 9 9}$ & 1.73848 & $\mathbf{1 . 9 6}$ & 2.17311 & $\mathbf{2 . 5 7}$ \\
\hline $\mathbf{2 2}$ & 3.02234 & $\mathbf{3 . 4 1}$ & 1.9523 & $\mathbf{1 . 8 6}$ & 2.49993 & $\mathbf{1 . 6 6}$ \\
\hline $\mathbf{2 3}$ & 3.39537 & $\mathbf{3 . 2 2}$ & 1.88156 & $\mathbf{2 . 1 4}$ & 1.67001 & $\mathbf{1 . 7 9}$ \\
\hline $\mathbf{2 4}$ & 3.36091 & $\mathbf{5 . 0 5}$ & 2.10766 & $\mathbf{1 . 7 2}$ & 1.82388 & $\mathbf{2 . 2 3}$ \\
\hline $\mathbf{2 5}$ & 4.93373 & $\mathbf{3 . 5 4}$ & 1.72616 & $\mathbf{1 . 8 0}$ & 2.27774 & $\mathbf{2 . 8 5}$ \\
\hline $\mathbf{2 6}$ & 3.54308 & $\mathbf{3 . 5 8}$ & 1.84928 & $\mathbf{2 . 4 4}$ & 2.82151 & $\mathbf{2 . 4 8}$ \\
\hline $\mathbf{2 7}$ & 3.51609 & $\mathbf{2 . 7 5}$ & 2.43307 & $\mathbf{2 . 3 5}$ & 2.44304 & $\mathbf{2 . 0 0}$ \\
\hline $\mathbf{2 8}$ & 2.71997 & $\mathbf{2 . 3 6}$ & 2.42315 & $\mathbf{3 . 3 0}$ & 2.04158 & $\mathbf{2 . 5 4}$ \\
\hline $\mathbf{2 9}$ & 2.38464 & $\mathbf{2 . 6 8}$ & 3.24533 & $\mathbf{2 . 5 9}$ & 2.54847 & $\mathbf{2 . 6 5}$ \\
\hline
\end{tabular}




\begin{tabular}{|l|l|l|l|l|l|l|}
$\mathbf{3 0}$ & 2.74468 & $\mathbf{3 . 5 2}$ & 2.55689 & $\mathbf{2 . 1 6}$ & 2.6962 & $\mathbf{3 . 2 5}$ \\
\hline $\mathbf{3 1}$ & & & 2.20851 & $\mathbf{2 . 7 9}$ & & \\
\hline
\end{tabular}

\begin{tabular}{|c|c|c|c|c|c|c|}
\hline \multicolumn{7}{|c|}{ PTTCH FORECAST TABLE $(\mathrm{n}=28$, and $\alpha=0.077)$} \\
\hline DAY & APRIL & & MAY & & JUNE & \\
\hline 1 & 97.56 & 97.56 & 97.89928 & 98.49 & 98.12847 & 98.23 \\
\hline 2 & 97.63007 & 98.47 & 98.46767 & 98.2 & 98.21075 & 97.98 \\
\hline 3 & 98.43997 & 98.08 & 98.21078 & 98.34 & 97.96152 & 97.74 \\
\hline 4 & 98.1262 & 98.68 & 98.32614 & 98.16 & 97.75232 & 97.90 \\
\hline 5 & 98.67307 & 98.59 & 98.22853 & 99.05 & 97.93619 & 98.37 \\
\hline 6 & 98.61849 & 98.96 & 98.9807 & 98.15 & 98.27452 & 97.13 \\
\hline 7 & 98.90302 & 98.22 & 98.12459 & 97.82 & 97.2301 & 98.43 \\
\hline 8 & 98.22385 & 98.27 & 97.05616 & 87.9 & 98.3915 & 97.93 \\
\hline 9 & 98.32852 & 99.03 & 88.64228 & 97.54 & 97.98313 & 98.62 \\
\hline 10 & 99.0146 & 98.83 & 97.58389 & 98.11 & 98.59536 & 98.30 \\
\hline 11 & 98.76455 & 97.98 & 98.07458 & 97.65 & 98.27844 & 98.02 \\
\hline 12 & 98.05623 & 98.97 & 97.73855 & 98.8 & 98.06543 & 98.61 \\
\hline 13 & 98.9469 & 98.67 & 98.77382 & 98.46 & 98.54455 & 97.76 \\
\hline 14 & 98.59223 & 97.66 & 98.42997 & 98.07 & 97.76 & 97.76 \\
\hline 15 & 97.7447 & 98.76 & 98.08463 & 98.26 & 97.84162 & 98.82 \\
\hline 16 & 98.74999 & 98.63 & 98.25769 & 98.23 & 98.79767 & 98.53 \\
\hline 17 & 98.57995 & 97.98 & 98.26311 & 98.66 & 98.48765 & 97.98 \\
\hline 18 & 97.96999 & 97.85 & 98.64768 & 98.5 & 97.96691 & 97.81 \\
\hline 19 & 97.8346 & 97.65 & 98.51925 & 98.75 & 97.80461 & 97.74 \\
\hline 20 & 97.70621 & 98.38 & 98.72074 & 98.37 & 97.78235 & 98.29 \\
\hline 21 & 98.28375 & 97.13 & 98.23448 & 96.61 & 98.136 & 96.29 \\
\hline 22 & 97.10767 & 96.84 & 96.76246 & 98.59 & 96.43091 & 98.12 \\
\hline 23 & 96.91315 & 97.79 & 98.57306 & 98.37 & 98.15311 & 98.55 \\
\hline 24 & 97.55207 & 94.7 & 98.38386 & $\mathbf{9 8 . 5 5}$ & 98.55 & 98.55 \\
\hline 25 & 94.93023 & 97.69 & 98.4037 & 96.65 & 98.2651 & 94.85 \\
\hline 26 & 97.57912 & 96.25 & 96.78321 & 98.38 & 95.09871 & 98.08 \\
\hline 27 & 96.43172 & 98.61 & 98.36845 & 98.23 & 98.07307 & 97.99 \\
\hline 28 & 98.47063 & 96.8 & 98.19997 & 97.84 & 97.99 & 97.99 \\
\hline 29 & 96.89394 & 98.02 & 97.82999 & 97.71 & 97.18689 & 87.56 \\
\hline 30 & 98.00691 & 97.85 & 97.22413 & 91.4 & 88.34694 & 97.78 \\
\hline
\end{tabular}

\begin{tabular}{|r|r|r|l|l|l|r|}
\hline \multicolumn{7}{|c|}{ PTFAIL FORECAST TABLE $(n=28$, and $\boldsymbol{\alpha}=\mathbf{0 . 0 7 7})$} \\
\hline DAY & APRIL & & MAY & & JUNE & \\
\hline $\mathbf{1}$ & 1.06 & $\mathbf{1 . 0 6}$ & 1.03078 & $\mathbf{1 . 1 6}$ & 1.10616 & $\mathbf{1 . 1 8}$ \\
\hline $\mathbf{2}$ & 1.06077 & $\mathbf{1 . 0 7}$ & 1.16616 & $\mathbf{1 . 2 4}$ & 1.18924 & $\mathbf{1 . 3}$ \\
\hline $\mathbf{3}$ & 1.06538 & $\mathbf{1 . 0 1}$ & 1.23846 & $\mathbf{1 . 2 2}$ & 1.31001 & $\mathbf{1 . 4 3}$ \\
\hline $\mathbf{4}$ & 1.01 & $\mathbf{1 . 0 1}$ & 1.22462 & $\mathbf{1 . 2 8}$ & 1.43308 & $\mathbf{1 . 4 7}$ \\
\hline $\mathbf{5}$ & 1.00923 & $\mathbf{1}$ & 1.26922 & $\mathbf{1 . 1 4}$ & 1.45229 & $\mathbf{1 . 2 4}$ \\
\hline $\mathbf{6}$ & 0.99615 & $\mathbf{0 . 9 5}$ & 1.1323 & $\mathbf{1 . 0 4}$ & 1.23846 & $\mathbf{1 . 2 2}$ \\
\hline
\end{tabular}




\begin{tabular}{|r|r|r|r|r|r|r|}
$\mathbf{7}$ & 0.95 & $\mathbf{0 . 9 5}$ & 1.04616 & $\mathbf{1 . 1 2}$ & 1.23386 & $\mathbf{1 . 4 0}$ \\
\hline $\mathbf{8}$ & 0.95539 & $\mathbf{1 . 0 2}$ & 1.10999 & $\mathbf{0 . 9 9}$ & 1.38152 & $\mathbf{1 . 1 6}$ \\
\hline $\mathbf{9}$ & 1.02462 & $\mathbf{1 . 0 8}$ & 0.9977 & $\mathbf{1 . 0 9}$ & 1.15923 & $\mathbf{1 . 1 5}$ \\
\hline $\mathbf{1 0}$ & 1.07615 & $\mathbf{1 . 0 3}$ & 1.08461 & $\mathbf{1 . 0 2}$ & 1.16078 & $\mathbf{1 . 2 9}$ \\
\hline $\mathbf{1 1}$ & 1.03077 & $\mathbf{1 . 0 4}$ & 1.02 & $\mathbf{1 . 0 2}$ & 1.28307 & $\mathbf{1 . 2}$ \\
\hline $\mathbf{1 2}$ & 1.04231 & $\mathbf{1 . 0 7}$ & 1.01538 & $\mathbf{0 . 9 6}$ & 1.19153 & $\mathbf{1 . 0 9}$ \\
\hline $\mathbf{1 3}$ & 1.06615 & $\mathbf{1 . 0 2}$ & 0.95846 & $\mathbf{0 . 9 4}$ & 1.09847 & $\mathbf{1 . 2}$ \\
\hline $\mathbf{1 4}$ & 1.02 & $\mathbf{1 . 0 2}$ & 0.94693 & $\mathbf{1 . 0 3}$ & 1.20308 & $\mathbf{1 . 2 4}$ \\
\hline $\mathbf{1 5}$ & 1.01692 & $\mathbf{0 . 9 8}$ & 1.02692 & $\mathbf{0 . 9 9}$ & 1.22768 & $\mathbf{1 . 0 8}$ \\
\hline $\mathbf{1 6}$ & 0.99309 & $\mathbf{1 . 1 5}$ & 0.98923 & $\mathbf{0 . 9 8}$ & 1.08462 & $\mathbf{1 . 1 4}$ \\
\hline $\mathbf{1 7}$ & 1.15539 & $\mathbf{1 . 2 2}$ & 0.99232 & $\mathbf{1 . 1 4}$ & 1.13923 & $\mathbf{1 . 1 3}$ \\
\hline $\mathbf{1 8}$ & 1.21076 & $\mathbf{1 . 1}$ & 1.14 & $\mathbf{1 . 1 4}$ & 1.13462 & $\mathbf{1 . 1 9}$ \\
\hline $\mathbf{1 9}$ & 1.10154 & $\mathbf{1 . 1 2}$ & 1.13923 & $\mathbf{1 . 1 3}$ & 1.20001 & $\mathbf{1 . 3 2}$ \\
\hline $\mathbf{2 0}$ & 1.11923 & $\mathbf{1 . 1 1}$ & 1.12307 & $\mathbf{1 . 0 4}$ & 1.31538 & $\mathbf{1 . 2 6}$ \\
\hline $\mathbf{2 1}$ & 1.10384 & $\mathbf{1 . 0 3}$ & 1.04077 & $\mathbf{1 . 0 5}$ & 1.25076 & $\mathbf{1 . 1 4}$ \\
\hline $\mathbf{2 2}$ & 1.02923 & $\mathbf{1 . 0 2}$ & 1.03306 & $\mathbf{0 . 8 3}$ & 1.14385 & $\mathbf{1 . 1 9}$ \\
\hline $\mathbf{2 3}$ & 1.02693 & $\mathbf{1 . 1 1}$ & 0.83693 & $\mathbf{0 . 9 2}$ & 1.18307 & $\mathbf{1 . 1}$ \\
\hline $\mathbf{2 4}$ & 1.11231 & $\mathbf{1 . 1 4}$ & 0.91769 & $\mathbf{0 . 8 9}$ & 1.09769 & $\mathbf{1 . 0 7}$ \\
\hline $\mathbf{2 5}$ & 1.13461 & $\mathbf{1 . 0 7}$ & 0.9054 & $\mathbf{1 . 0 9}$ & 1.07693 & $\mathbf{1 . 1 6}$ \\
\hline $\mathbf{2 6}$ & 1.07231 & $\mathbf{1 . 1}$ & 1.08461 & $\mathbf{1 . 0 2}$ & 1.15923 & $\mathbf{1 . 1 5}$ \\
\hline $\mathbf{2 7}$ & 1.09384 & $\mathbf{1 . 0 2}$ & 1.03232 & $\mathbf{1 . 1 8}$ & 1.15539 & $\mathbf{1 . 2 2}$ \\
\hline $\mathbf{2 8}$ & 1.02308 & $\mathbf{1 . 0 6}$ & 1.17923 & $\mathbf{1 . 1 7}$ & 1.21692 & $\mathbf{1 . 1 8}$ \\
\hline $\mathbf{2 9}$ & 1.05538 & $\mathbf{1}$ & 1.18309 & $\mathbf{1 . 3 4}$ & 1.18231 & $\mathbf{1 . 2 1}$ \\
\hline $\mathbf{3 0}$ & 1.00154 & $\mathbf{1 . 0 2}$ & 1.32999 & $\mathbf{1 . 2 1}$ & 1.21847 & $\mathbf{1 . 3 2}$ \\
\hline $\mathbf{3 1}$ & & & 1.20153 & $\mathbf{1 . 1}$ & & \\
\hline & & & & & & \\
\hline
\end{tabular}

\begin{tabular}{|r|r|r|r|r|r|r|}
\hline \multicolumn{6}{|c|}{ PCDROP FORECAST TABLE $(\mathbf{n}=\mathbf{2 8}$, and $\boldsymbol{\alpha}=\mathbf{0 . 0 7 7})$} \\
\hline DAY & \multicolumn{1}{|c|}{ APRIL } & & \multicolumn{1}{l|}{ MAY } & & \multicolumn{1}{|l|}{ JUNE } & \\
\hline $\mathbf{1}$ & 0.40012 & $\mathbf{0 . 4}$ & 0.36154 & $\mathbf{0 . 3 8}$ & 0.35 & $\mathbf{0 . 3 5}$ \\
\hline $\mathbf{2}$ & 0.40077 & $\mathbf{0 . 4 1}$ & 0.38154 & $\mathbf{0 . 4}$ & 0.35462 & $\mathbf{0 . 4 1}$ \\
\hline $\mathbf{3}$ & 0.40769 & $\mathbf{0 . 3 8}$ & 0.40077 & $\mathbf{0 . 4 1}$ & 0.40846 & $\mathbf{0 . 3 9}$ \\
\hline $\mathbf{4}$ & 0.38231 & $\mathbf{0 . 4 1}$ & 0.40846 & $\mathbf{0 . 3 9}$ & 0.39385 & $\mathbf{0 . 4 4}$ \\
\hline $\mathbf{5}$ & 0.40846 & $\mathbf{0 . 3 9}$ & 0.38692 & $\mathbf{0 . 3 5}$ & 0.43769 & $\mathbf{0 . 4 1}$ \\
\hline $\mathbf{6}$ & 0.38769 & $\mathbf{0 . 3 6}$ & 0.35 & $\mathbf{0 . 3 5}$ & 0.40307 & $\mathbf{0 . 3 2}$ \\
\hline $\mathbf{7}$ & 0.36154 & $\mathbf{0 . 3 8}$ & 0.35847 & $\mathbf{0 . 4 6}$ & 0.3277 & $\mathbf{0 . 4 2}$ \\
\hline $\mathbf{8}$ & 0.37923 & $\mathbf{0 . 3 7}$ & 0.46154 & $\mathbf{0 . 4 8}$ & 0.41384 & $\mathbf{0 . 3 4}$ \\
\hline $\mathbf{9}$ & 0.37077 & $\mathbf{0 . 3 8}$ & 0.47307 & $\mathbf{0 . 3 9}$ & 0.33846 & $\mathbf{0 . 3 2}$ \\
\hline $\mathbf{1 0}$ & 0.37923 & $\mathbf{0 . 3 7}$ & 0.38846 & $\mathbf{0 . 3 7}$ & 0.33232 & $\mathbf{0 . 4 8}$ \\
\hline $\mathbf{1 1}$ & 0.37462 & $\mathbf{0 . 4 3}$ & 0.37077 & $\mathbf{0 . 3 8}$ & 0.47846 & $\mathbf{0 . 4 6}$ \\
\hline $\mathbf{1 2}$ & 0.42846 & $\mathbf{0 . 4 1}$ & 0.38 & $\mathbf{0 . 3 8}$ & 0.45307 & $\mathbf{0 . 3 7}$ \\
\hline $\mathbf{1 3}$ & 0.40923 & $\mathbf{0 . 4}$ & 0.38231 & $\mathbf{0 . 4 1}$ & 0.36923 & $\mathbf{0 . 3 6}$ \\
\hline $\mathbf{1 4}$ & 0.40154 & $\mathbf{0 . 4 2}$ & 0.41077 & $\mathbf{0 . 4 2}$ & 0.37309 & $\mathbf{0 . 5 3}$ \\
\hline $\mathbf{1 5}$ & 0.42077 & $\mathbf{0 . 4 3}$ & 0.41846 & $\mathbf{0 . 4}$ & 0.53 & $\mathbf{0 . 5 3}$ \\
\hline $\mathbf{1 6}$ & 0.42846 & $\mathbf{0 . 4 1}$ & 0.40385 & $\mathbf{0 . 4 5}$ & 0.5223 & $\mathbf{0 . 4 3}$ \\
\hline
\end{tabular}




\begin{tabular}{|r|r|r|r|r|r|r|}
$\mathbf{1 7}$ & 0.40846 & $\mathbf{0 . 3 9}$ & 0.44615 & $\mathbf{0 . 4}$ & 0.43231 & $\mathbf{0 . 4 6}$ \\
\hline $\mathbf{1 8}$ & 0.39231 & $\mathbf{0 . 4 2}$ & 0.39846 & $\mathbf{0 . 3 8}$ & 0.46385 & $\mathbf{0 . 5 1}$ \\
\hline $\mathbf{1 9}$ & 0.42 & $\mathbf{0 . 4 2}$ & 0.38077 & $\mathbf{0 . 3 9}$ & 0.51154 & $\mathbf{0 . 5 3}$ \\
\hline $\mathbf{2 0}$ & 0.42077 & $\mathbf{0 . 4 3}$ & 0.38846 & $\mathbf{0 . 3 7}$ & 0.52538 & $\mathbf{0 . 4 7}$ \\
\hline $\mathbf{2 1}$ & 0.42615 & $\mathbf{0 . 3 8}$ & 0.37154 & $\mathbf{0 . 3 9}$ & 0.47231 & $\mathbf{0 . 5}$ \\
\hline $\mathbf{2 2}$ & 0.38 & $\mathbf{0 . 3 8}$ & 0.38461 & $\mathbf{0 . 3 2}$ & 0.51001 & $\mathbf{0 . 6 3}$ \\
\hline $\mathbf{2 3}$ & 0.38231 & $\mathbf{0 . 4 1}$ & 0.32 & $\mathbf{0 . 3 2}$ & 0.61537 & $\mathbf{0 . 4 4}$ \\
\hline $\mathbf{2 4}$ & 0.41462 & $\mathbf{0 . 4 7}$ & 0.32462 & $\mathbf{0 . 3 8}$ & 0.43923 & $\mathbf{0 . 4 3}$ \\
\hline $\mathbf{2 5}$ & 0.46461 & $\mathbf{0 . 4}$ & 0.38 & $\mathbf{0 . 3 8}$ & 0.43385 & $\mathbf{0 . 4 8}$ \\
\hline $\mathbf{2 6}$ & 0.40077 & $\mathbf{0 . 4 1}$ & 0.37538 & $\mathbf{0 . 3 2}$ & 0.48308 & $\mathbf{0 . 5 2}$ \\
\hline $\mathbf{2 7}$ & 0.40615 & $\mathbf{0 . 3 6}$ & 0.32154 & $\mathbf{0 . 3 4}$ & 0.51769 & $\mathbf{0 . 4 9}$ \\
\hline $\mathbf{2 8}$ & 0.36462 & $\mathbf{0 . 4 2}$ & 0.35001 & $\mathbf{0 . 4 7}$ & 0.49616 & $\mathbf{0 . 5 7}$ \\
\hline $\mathbf{2 9}$ & 0.42154 & $\mathbf{0 . 4 4}$ & 0.47154 & $\mathbf{0 . 4 9}$ & 0.57077 & $\mathbf{0 . 5 8}$ \\
\hline $\mathbf{3 0}$ & 0.43384 & $\mathbf{0 . 3 6}$ & 0.48846 & $\mathbf{0 . 4 7}$ & 0.57461 & $\mathbf{0 . 5 1}$ \\
\hline $\mathbf{3 1}$ & & & 0.46076 & $\mathbf{0 . 3 5}$ & & \\
\hline
\end{tabular}

\begin{tabular}{|r|r|r|r|r|r|r|}
\hline \multicolumn{6}{|c|}{ PCCONG FORECAST TABLE $(\mathbf{n}=\mathbf{2 8}$, and $\boldsymbol{\alpha}=\mathbf{0 . 0 7 7})$} \\
\hline DAY & \multicolumn{1}{|c|}{ APRIL } & & \multicolumn{1}{l|}{ MAY } & & \multicolumn{1}{l|}{ JUNE } & \\
\hline $\mathbf{1}$ & 1.410976 & $\mathbf{1 . 4 1 0 9 7 6}$ & 2.34911 & $\mathbf{0 . 9}$ & 1.17461 & $\mathbf{1 . 1 1}$ \\
\hline $\mathbf{2}$ & 1.38998 & $\mathbf{1 . 1 5}$ & 0.90616 & $\mathbf{0 . 9 8}$ & 1.1023 & $\mathbf{1 . 0 1}$ \\
\hline $\mathbf{3}$ & 1.13691 & $\mathbf{0 . 9 8}$ & 0.97076 & $\mathbf{0 . 8 6}$ & 0.99691 & $\mathbf{0 . 8 4}$ \\
\hline $\mathbf{4}$ & 0.95998 & $\mathbf{0 . 7 2}$ & 0.83151 & $\mathbf{0 . 4 9}$ & 0.83769 & $\mathbf{0 . 8 1}$ \\
\hline $\mathbf{5}$ & 0.70922 & $\mathbf{0 . 5 8}$ & 0.50309 & $\mathbf{0 . 6 6}$ & 0.79383 & $\mathbf{0 . 6}$ \\
\hline $\mathbf{6}$ & 0.55844 & $\mathbf{0 . 3}$ & 0.66154 & $\mathbf{0 . 6 8}$ & 0.60462 & $\mathbf{0 . 6 6}$ \\
\hline $\mathbf{7}$ & 0.31001 & $\mathbf{0 . 4 3}$ & 0.69694 & $\mathbf{0 . 9}$ & 0.64614 & $\mathbf{0 . 4 8}$ \\
\hline $\mathbf{8}$ & 0.45387 & $\mathbf{0 . 7 4}$ & 1.5237 & $\mathbf{9}$ & 0.49463 & $\mathbf{0 . 6 7}$ \\
\hline $\mathbf{9}$ & 0.72999 & $\mathbf{0 . 6 1}$ & 8.37168 & $\mathbf{0 . 8 4}$ & 0.68309 & $\mathbf{0 . 8 4}$ \\
\hline $\mathbf{1 0}$ & 0.60153 & $\mathbf{0 . 5}$ & 0.81921 & $\mathbf{0 . 5 7}$ & 0.88774 & $\mathbf{1 . 4 6}$ \\
\hline $\mathbf{1 1}$ & 0.58932 & $\mathbf{1 . 6 6}$ & 0.58617 & $\mathbf{0 . 7 8}$ & 1.47386 & $\mathbf{1 . 6 4}$ \\
\hline $\mathbf{1 2}$ & 1.58377 & $\mathbf{0 . 6 7}$ & 0.73996 & $\mathbf{0 . 2 6}$ & 1.60458 & $\mathbf{1 . 1 8}$ \\
\hline $\mathbf{1 3}$ & 0.6469 & $\mathbf{0 . 3 7}$ & 0.26308 & $\mathbf{0 . 3}$ & 1.20926 & $\mathbf{1 . 5 6}$ \\
\hline $\mathbf{1 4}$ & 0.52631 & $\mathbf{2 . 4}$ & 0.33927 & $\mathbf{0 . 8 1}$ & 1.51611 & $\mathbf{0 . 9 9}$ \\
\hline $\mathbf{1 5}$ & 2.24369 & $\mathbf{0 . 3 7}$ & 0.79075 & $\mathbf{0 . 5 6}$ & 0.9746 & $\mathbf{0 . 7 9}$ \\
\hline $\mathbf{1 6}$ & 0.39926 & $\mathbf{0 . 7 5}$ & 0.57001 & $\mathbf{0 . 6 9}$ & 0.79 & $\mathbf{0 . 7 9}$ \\
\hline $\mathbf{1 7}$ & 0.79543 & $\mathbf{1 . 3 4}$ & 0.68846 & $\mathbf{0 . 6 7}$ & 0.82003 & $\mathbf{1 . 1 8}$ \\
\hline $\mathbf{1 8}$ & 1.30535 & $\mathbf{0 . 8 9}$ & 0.67077 & $\mathbf{0 . 6 8}$ & 1.22466 & $\mathbf{1 . 7 6}$ \\
\hline $\mathbf{1 9}$ & 0.87922 & $\mathbf{0 . 7 5}$ & 0.68924 & $\mathbf{0 . 8}$ & 1.70918 & $\mathbf{1 . 1}$ \\
\hline $\mathbf{2 0}$ & 0.77618 & $\mathbf{1 . 0 9}$ & 0.76843 & $\mathbf{0 . 3 9}$ & 1.08845 & $\mathbf{0 . 9 5}$ \\
\hline $\mathbf{2 1}$ & 1.08307 & $\mathbf{1}$ & 0.42388 & $\mathbf{0 . 8 3}$ & 1.00621 & $\mathbf{1 . 6 8}$ \\
\hline $\mathbf{2 2}$ & 1.00231 & $\mathbf{1 . 0 3}$ & 0.80998 & $\mathbf{0 . 5 7}$ & 1.61994 & $\mathbf{0 . 9}$ \\
\hline $\mathbf{2 3}$ & 1.07081 & $\mathbf{1 . 5 6}$ & 0.5623 & $\mathbf{0 . 4 7}$ & 0.87613 & $\mathbf{0 . 5 9}$ \\
\hline $\mathbf{2 4}$ & 1.91343 & $\mathbf{6 . 1 5}$ & 0.48771 & $\mathbf{0 . 7}$ & 0.61464 & $\mathbf{0 . 9 1}$ \\
\hline $\mathbf{2 5}$ & 5.7727 & $\mathbf{1 . 2 5}$ & 0.74158 & $\mathbf{1 . 2 4}$ & 1.08941 & $\mathbf{3 . 2 4}$ \\
\hline $\mathbf{2 6}$ & 1.25385 & $\mathbf{1 . 3}$ & 1.22691 & $\mathbf{1 . 0 7}$ & 3.10448 & $\mathbf{1 . 4 8}$ \\
\hline & & & & & & \\
\hline
\end{tabular}




\begin{tabular}{|r|r|r|r|r|r|r|}
$\mathbf{2 7}$ & 1.28537 & $\mathbf{1 . 1 1}$ & 1.05845 & $\mathbf{0 . 9 2}$ & 1.44381 & $\mathbf{1 . 0 1}$ \\
\hline $\mathbf{2 8}$ & 1.10153 & $\mathbf{1}$ & 0.95542 & $\mathbf{1 . 3 8}$ & 1.00384 & $\mathbf{0 . 9 3}$ \\
\hline $\mathbf{2 9}$ & 0.97151 & $\mathbf{0 . 6 3}$ & 1.37615 & $\mathbf{1 . 3 3}$ & 0.97389 & $\mathbf{1 . 5}$ \\
\hline $\mathbf{3 0}$ & 0.77168 & $\mathbf{2 . 4 7}$ & 1.3839 & $\mathbf{2 . 0 3}$ & 1.50462 & $\mathbf{1 . 5 6}$ \\
\hline $\mathbf{3 1}$ & & & 1.96455 & $\mathbf{1 . 1 8}$ & & \\
\hline
\end{tabular}

\begin{tabular}{|c|c|c|c|c|c|c|}
\hline \multicolumn{7}{|c|}{ PCFAIL FORECAST TABLE $(\mathrm{n}=28$, and $\alpha=0.077)$} \\
\hline DAY & APRIL & & MAY & & JUNE & \\
\hline 1 & 2.982104 & 2.982104 & 4.58524 & 2.85 & 2.70384 & 2.63 \\
\hline 2 & 2.97384 & 2.9 & 2.84692 & 2.81 & 2.67774 & 3.25 \\
\hline 3 & 2.88229 & 2.67 & 2.81 & 2.81 & 3.21073 & 2.74 \\
\hline 4 & 2.68463 & 2.86 & 2.81693 & 2.9 & 2.86089 & 4.31 \\
\hline 5 & 2.84075 & 2.61 & 2.92926 & 3.28 & 4.22915 & 3.26 \\
\hline 6 & 2.61077 & 2.62 & 3.24997 & 2.89 & 3.2138 & 2.66 \\
\hline 7 & 2.62308 & 2.66 & 2.93081 & 3.42 & 2.6831 & 2.96 \\
\hline 8 & 2.66154 & 2.68 & 3.44002 & 3.68 & 2.95076 & 2.84 \\
\hline 9 & 2.68693 & 2.77 & 3.62148 & 2.92 & 2.82306 & 2.62 \\
\hline 10 & 2.7623 & 2.67 & 2.92462 & 2.98 & 2.73011 & 4.05 \\
\hline 11 & 2.75393 & 3.76 & 2.9723 & 2.88 & 3.97685 & 3.1 \\
\hline 12 & 3.69994 & 2.98 & 2.85228 & 2.52 & 3.07074 & 2.72 \\
\hline 13 & 3.00079 & 3.25 & 2.5508 & 2.92 & 2.71461 & 2.65 \\
\hline 14 & 3.45251 & 5.88 & 2.9277 & 3.02 & 2.68388 & 3.09 \\
\hline 15 & 5.6798 & 3.28 & 3.02693 & 3.11 & 3.09616 & 3.17 \\
\hline 16 & 3.25228 & 2.92 & 3.10846 & 3.09 & 3.16615 & 3.12 \\
\hline 17 & 2.92616 & 3 & 3.07383 & 2.88 & 3.12385 & 3.17 \\
\hline 18 & 2.97921 & 2.73 & 2.89155 & 3.03 & 3.17231 & 3.2 \\
\hline 19 & 2.79083 & 3.52 & 3.00459 & 2.7 & 3.21925 & 3.45 \\
\hline 20 & 3.49074 & 3.14 & 2.69076 & 2.58 & 3.45308 & 3.49 \\
\hline 21 & 3.13307 & 3.05 & 2.58385 & 2.63 & 3.46844 & 3.21 \\
\hline 22 & 3.08542 & 3.51 & 2.63 & 2.63 & 3.29008 & 4.25 \\
\hline 23 & 3.50615 & 3.46 & 2.6223 & 2.53 & 4.15144 & 2.97 \\
\hline 24 & 3.60168 & 5.3 & 2.53539 & 2.6 & 2.96923 & 2.96 \\
\hline 25 & 5.20452 & 4.06 & 2.60462 & 2.66 & 3.0293 & 3.86 \\
\hline 26 & 3.96221 & 2.79 & 2.68002 & 2.92 & 3.8138 & 3.26 \\
\hline 27 & 2.81849 & 3.16 & 2.88381 & 2.45 & 3.25615 & 3.21 \\
\hline 28 & 3.15153 & $\mathbf{3 . 0 5}$ & 2.47387 & 2.76 & 3.22386 & 3.39 \\
\hline 29 & 3.03999 & 2.92 & 2.77386 & 2.94 & 3.37768 & 3.23 \\
\hline 30 & 3.05937 & 4.73 & 2.92537 & 2.75 & 3.25695 & 3.58 \\
\hline 31 & & & 2.74692 & 2.71 & & \\
\hline
\end{tabular}

